\title{
Non periodic patterning of super-hydrophobic surfaces for the manipulation of few molecules
}

\author{
F. Gentile ${ }^{\mathrm{a}, \mathrm{b}, *}$, M.L. Coluccio a ${ }^{\mathrm{a}}$ E. Rondanina ${ }^{\mathrm{b}}$, S. Santoriello ${ }^{\mathrm{a}}$, D. Di Mascolo ${ }^{\mathrm{a}}$, A. Accardo ${ }^{\mathrm{b}}$, M. Francardi $^{\mathrm{a}}$, \\ F. De Angelis ${ }^{\mathrm{b}}$, P. Candeloro ${ }^{\mathrm{a}}$, E. Di Fabrizio ${ }^{\mathrm{a}, \mathrm{b}}$ \\ ${ }^{a}$ BioNEM (Bio Nano Engineering and Technology for Medicine), University Magna Graecia of Catanzaro - Catanzaro 88100, Italy \\ ${ }^{\mathrm{b}}$ Nanostructures Department, Italian Institute of Technology. Via Morego, 3016163 Genova, Italy
}

\section{A R T I C L E I N F O}

\section{Article history:}

Available online 8 February 2013

\section{Keywords:}

Super-hydrophobic Surfaces

SERS

SEIRA

Single molecule detection

Bio inspired materials

Mathematical representation of surfaces

\begin{abstract}
A B S T R A C T
Super-hydrophobic ( $\mathrm{SH}$ ) surfaces are bio-inspired, nanotechnology artifacts which feature a reduced friction coefficient whereby they can be used for a number of very practical applications including, on the medical side, the manipulation of biological solutions. These surfaces can be combined with bio-photonic devices to obtain an integrated lab-on-a-chip system where, on a first stage, the SH surface would vehicle or transport the analytes of interest into a small area and, on a second stage, the bio-sensors would permit, in that area, the detection of the solute with the resolution of a single molecule. This novel diagnostic modality offers realistic possibilities for the early detection of cancers. Nevertheless, as it stands, the device still suffers from the severe disadvantage that the exact final position of the solute, upon evaporation, is unpredictable, and thus the localization and recognition of few molecules would be impractical. Conventional SH surfaces typically comprise micro pillars combined to form a regular hexagonal motif. Here, the periodicity of those pillars was broken introducing artificial gradients of wettability over the surface. In doing so, some regions are rendered more hydrophilic than others and, on account of this, a solute would preferentially target these hydrophilic regions upon evaporation. In this work, such non regular geometries were realized and used to condense diluted Rhodamine solutions in a small area. Randomly distributed silver nano aggregates, conveniently positioned upon the micropillars, permitted the identification of few molecules using enhanced Fourier transform infrared spectroscopy (FTIR) spectroscopy.
\end{abstract}

(c) 2013 Elsevier B.V. All rights reserved.

\section{Introduction}

Bio sensors, and especially SERS/SEIRA (surface enhanced Raman spectroscopy/surface enhanced infra-red absorption) substrates, are capable to perform sensing measurements at femto- or atto molar sample concentrations with single molecule resolution. Detection limits down to the single-molecule level have been achieved, with potential applications ranging from the early diagnosis of diseases to the fast sequencing of genomes [1]. However, in many applications, transporting target molecules in extremely dilute solutions to these tiny sensors is a significant challenge

\footnotetext{
Abbreviations: SH, super-hydrophobic; SERS, surface enhanced Raman spectroscopy; SEIRA, surface enhanced infra-red absorption; 2D, two-dimensional; FTIR, Fourier transform infra red; SEM, scanning electron microscopy.

* Corresponding author at: BioNEM (Bio Nano Engineering and Technology for Medicine), University Magna Graecia of Catanzaro - Catanzaro 88100, Italy. Tel.: +390961369 5896; fax: +3909613695225.

E-mail addresses: gentile@unicz.it, francesco.gentile77@gmail.com (F. Gentile).
}

in that the process is limited by diffusion, and often involves impractical timescales [2].

Recently, novel devices debuted in the field of nanotechnology which promise to surpass the obstacle of diffusion [2-4]. Those devices are inspired by the lotus effect [5,6], whereby arrays of micropillars are artificially incorporated to silicon surfaces, rendering these super-hydrophobic. To boost the Raman signal further, a range of plasmonic structures was integrated into $\mathrm{SH}$ silicon pillars. These metal structures serve as hot spots that intensify the local electric fields and consequently enhance the Raman signal by several orders of magnitude. And thus on a larger micrometric scale, the surface brings the molecules in an ultralow-concentration droplet to the active sites of the device and, there, on a smaller nanometric scale, nanosensors would sense those molecules with otherwise unattainable sensitivity. The droplet evaporation time, which takes from a few seconds to several minutes depending on its size, dramatically shortens the long waiting time of hours or even days for the traditional diffusion process $[7,8]$. This concept was developed even further. In [9,10], polymer based SH surfaces and X-ray spectroscopy were used to probe the crystal structure 
of proteins; in [11,12], SH surfaces and nanoporous silicon matrices were combined to yield devices with the capability of concentrating and harvesting small molecules, where the cut-off size can be adequately controlled.

In all these configurations, the pattern of pillars is periodic, that is, the substrate can be obtained as a regular tiling of a primitive cell, where the cell is a basis for the lattice. The disadvantage of regular arrays of pillars, is that the final place where the analyte evaporates is basically unpredictable. In [2], the solute was enforced to evaporate in a determined point of the substrate by breaking the symmetry of the pattern, that is, introducing, in that point, a cone in place of a pillar. Here, another approach is proposed, where gradients of wettability are artificially introduced in the pattern of pillars. This is practically done realizing a nonperiodic array of micropillars, that is, a distribution where the pitch $\delta$ between the pillars is not constant, and it would instead smoothly transition from an external region, where $\delta$ is large, to an inner region, where $\delta$ is small (Fig. 1a). By doing so, the hydrophobicity would not be constant over the substrate, rather some regions would be more hydrophilic than others; on account of this augmented hydrophilicity, a solute, upon evaporation, would preferentially target these hydrophilic regions, which would therefore be the active sites of the devices, and, in these sites solely, superior nano sensors can be embedded. The non uniform profile of pillars can be obtained using a contraction, that is a mathematical operation that, on a metric space and with respect to a fixed point, would displace the pillars in a fashion that their mutual distance would vary following a certain law. The surface energy of the substrate can be therefore determined (Fig. 1b) and, on deriving that with respect to space, the non uniform field of forces exerted on the drop during evaporation (Fig. 1c). This has some similarity with the general theory of relativity, where mass warps space-time to create gravitational fields.
In this work, such non regular geometries were realized (Fig. 1d-e) and used to condense diluted $\left(c=10^{-16} \mathrm{M}\right)$ Rhodamine solutions in a small area. Randomly distributed silver nano aggregates (Fig. 1f), conveniently positioned upon the micropillars, permitted the identification of few molecules using enhanced FTIR spectroscopy (Fig. 2). This mathematical description of surfaces provides a tools for those interested in designing smart platforms for the manipulation and analysis of extremely diluted solutions.

The concept of utilizing solids decorated with a texture of variable density is not new. In [13], Reyssat and colleagues realized microtextured surfaces made with posts of variable spacing, providing a gradient of post density. Therefore, in impacting water drops and observing their direction of rebound, they furnished an explanation of why vibrated drops move on such surface. In [14], Spori and colleagues fabricated density gradients of randomly placed holes and pillars by means of photolithography, which are a useful tool for investigating the influence of structural effects on a variety of different wetting phenomena, such as static and dynamic contact angles and roll-off angle.

These approaches represent, themselves, significant advances in the field of super hydrophobic surfaces, especially because provide valuable insight into the physical mechanisms that control the formation of drops upon textures of variable density, moreover, they introduce a mathematical description of the phenomenon.

However, our work departs from these approaches at least for two aspects. Differently from the other described works, we have introduced a gradient of wettability in two different directions, rather the one, thus providing a complete, bi-dimensional description of the pillars density in the plane. To these extents, our scheme is more general or unspecific, in that it can be applied to interpret, illustrate, or resolve a great variety of very practical situations, including the development and design of devices for the precise localization of a solute. This framework is not only conceptual or (a)

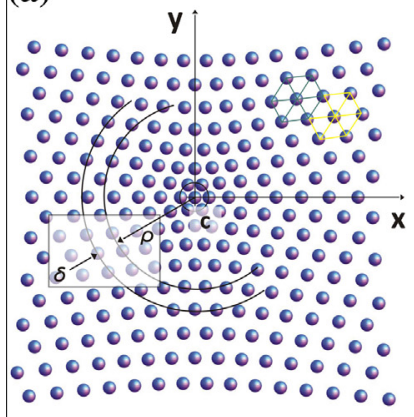

(d)

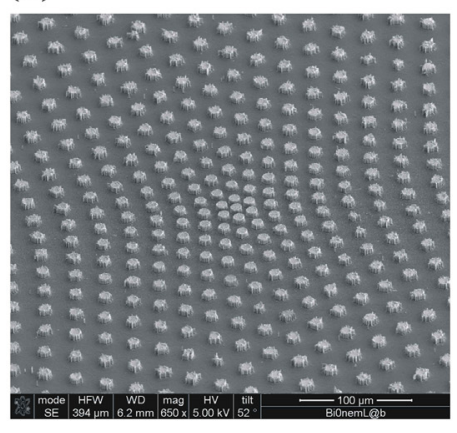

$50 \mu \mathrm{m}$ (b)

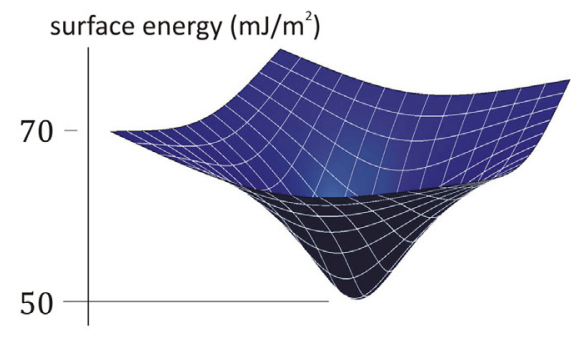

(c)

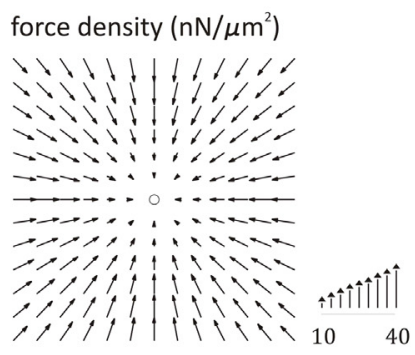

(f)

(e)

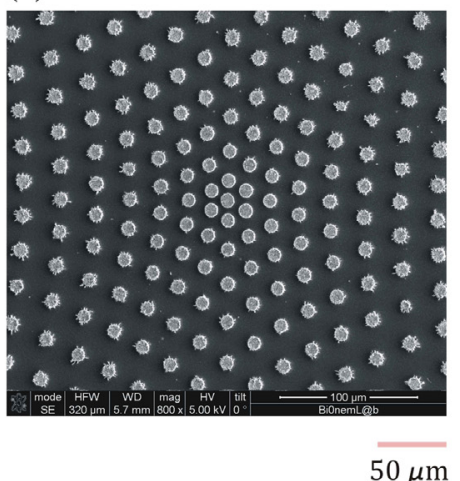

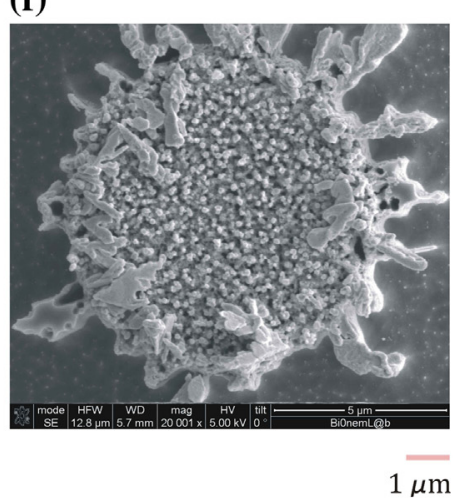

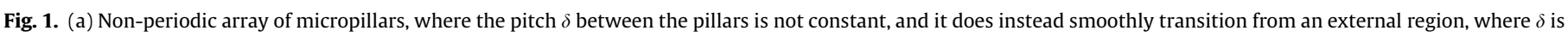

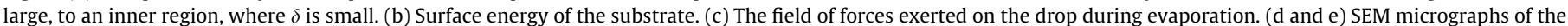

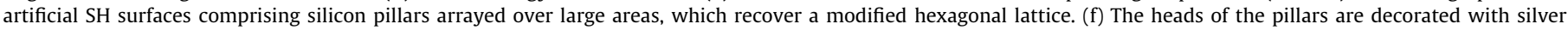
nanograin clusters. 
(a)

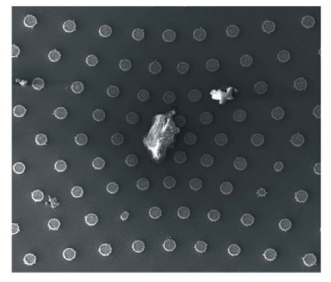

$10 \mu \mathrm{m}$

(d)

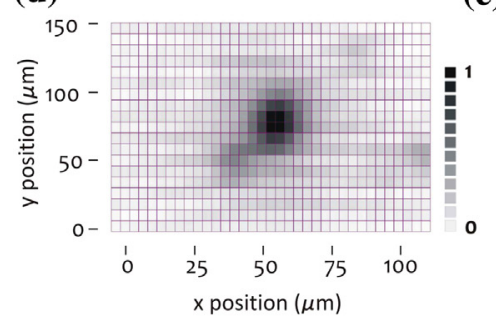

(b)

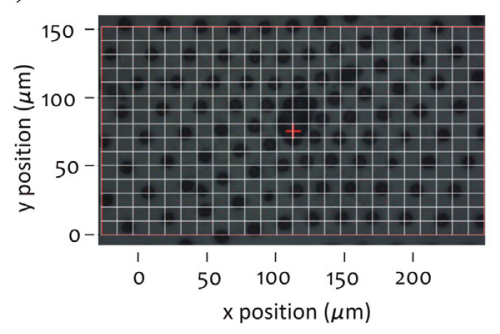

(e)

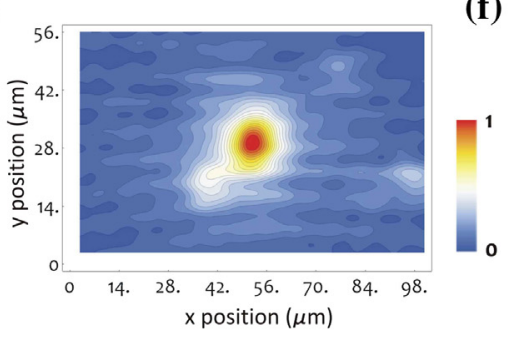

(c)

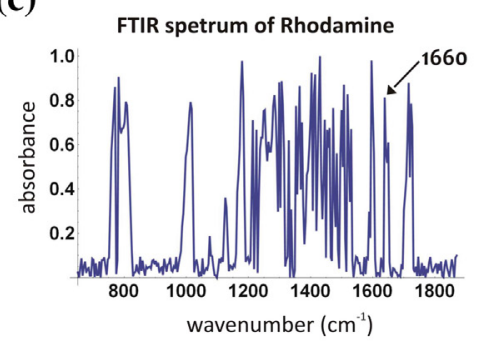

(f)

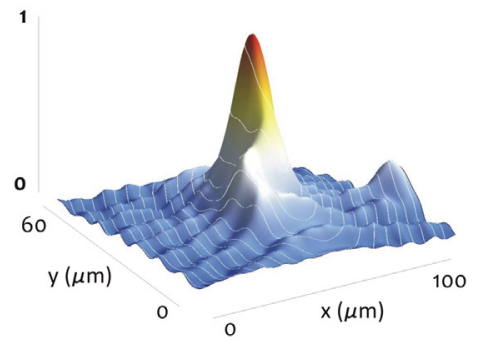

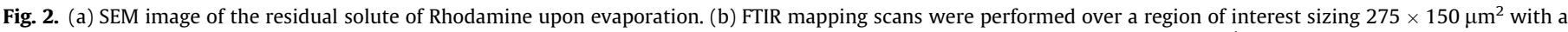

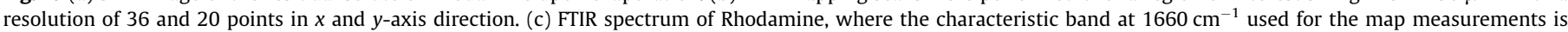

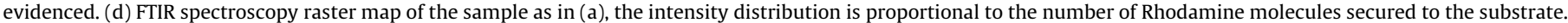
(e and f) A polynomial curve fitting of order three was used to derive the interpolated 3D and contour maps with sub-pixel resolution.

a theoretic exercise, in fact, it can be integrated into multifunctional devices and utilized to detect molecules in exceptionally law concentration ranges.

\section{Materials and methods}

SH-surfaces were designed and micro fabricated. These comprise micro pillars combined to form a modified hexagonal motif (Fig. 1d-e); silver nanoparticles (Fig. 1f) were distributed upon the pillars to guarantee superior SEIRA capabilities [15]. These devices are super-hydrophobic on account of their hybrid nature. The micro (that are, the pillars) and the nano (that is, the nanoparticles ensemble) scales combine hierarchically to yield non-wetting properties, as explained in $[3,16]$.

Small drops $(R \sim 1 \mathrm{~mm})$ of D.I. water containing Rhodamine in a low concentration $\left(10^{-16} \mathrm{M}\right)$ were gently positioned upon the surfaces and left evaporating over time. At the end of the process, few molecules were accumulated in a small region of the plane. The evaporation was performed at room temperature in a clean room to reduce the presence of external contaminants, and lasted approximately $30 \mathrm{~min}$. Upon evaporation, the residual solute was observed using direct SEM, and SIERA/FTIR spectroscopy techniques.

Rhodamine (R6G) was purchased from Sigma. Rhodamine6G is an organic compound and is used extensively in biotechnology applications. De-ionized (D.I.) water (Milli-Q Direct 3, Millipore) was used for all experiments. All chemicals, unless mentioned elsewhere, were of analytical grade and were used as received.

\subsection{Mathematical description of surfaces}

Consider an initial configuration where the pillars are arrayed to form a regular hexagonal lattice; in this framework, the diameter $\mathrm{d}$ of the pillars is $10 \mu \mathrm{m}$, the pitch $\delta_{0}$ is $30 \mu \mathrm{m}$ and, accordingly, the fraction of solid $\phi_{0}=\pi / 4\left(d / \delta_{0}\right)^{2}$ derived being $\pi / 36 \sim 0.1$. Notice that these parameters are consistent with a criterion of optimal design as described, for instance, in [2]. This pattern is then conveniently modified to incorporate a non uniform distribution of pillars. To do this, one can use a contraction $\chi$, that is a mathemat- ical, non dimensional operation that, on a metric space and with respect to a fixed point, would displace the pillars in a fashion that their mutual distance would vary following a certain law. It is convenient here adopting a polar coordinate system as in Fig. 1a and, in this frame of reference, $\chi$ takes the general form

$\chi(\rho)=A+B(\rho / l)^{\varepsilon}$

where $\rho=\left(x^{2}+y^{2}\right)^{1 / 2}$ is the radial distance from the contraction fixed point $c=\left(c_{x}, c_{y}\right), x$ and $y$ are the orthogonal Cartesian coordinates of the 2D manifold with origin $c, l=400 \mu \mathrm{m}$ is a characteristic length that defines the region of the plane where the contraction mapping is effective, and $A, B$ and $\varepsilon$ are constants that one can adjust at will to obtain a specific pillars profile and, for the present configuration, $A=0, B=1$ and $\varepsilon=1 / 3$. Therefore, the contraction $\chi$ is a scale operation that, for each pillar in the plane, assigns to that pillar a new position vector $r \rightarrow \chi r$, where $\chi<1$, and where the degree of this substitution depends not linearly on $\rho$. This implies that the distance between those pillars, that is the pitch $\delta$, is no more constant, and instead varies as a function of the distance $\rho$ from the fixed point $c$. $c$ can practically coincide with the center of one pillar, and thus that pillar would be the origin of the new coordinate system, and the spot where all the solute would preferentially concentrate upon evaporation.

Assuming a small initial inter particle distance $\delta_{0}$ (that is a reasonable hypothesis, since we are working at the micro scales), the revised pitch $\delta$ generated upon the application of the operation of contraction can be derived as

$\delta(\rho)=\delta_{0}(1-\delta \chi / \delta \rho)$,

and, on inverting Eq. (2), one can determine which is the correct contraction $\chi$ to apply to the lattice in order to obtain the desired pitch $\delta$

$\chi(\rho)=\int\left(\delta_{0}-\delta(\rho)\right) / \delta_{0} d \rho$

Once that $\chi$ is determined, the calculus of the modified solid fraction $\phi$ is trivial, being $\phi=\chi \phi_{0}$ and, from this, the surface energy of the substrate can be derived (Fig. 1b), with [17], as the weighted average of the surface energies at the water-solid, water-air interfaces, where the weights are $\phi$ and $(1-\phi)$ : 
$\sigma(\rho)=53 \phi(\rho)+72(1-\phi(\rho)) m l / m^{2}$

and notice that the minimum of energy is found in correspondence of $c$, that is also the most hydrophilic point in the modified lattice of pillars. The surface energy $\sigma$ can be further derived with respect to the spatial Cartesian coordinates and, in doing so, the non uniform field of forces exerted on the drop during evaporation can be estimated (Fig. 1c)

$T(x, y)=-(\partial / \partial x, \partial / \partial y) \sigma=-\nabla \sigma$

Remarkably, this procedure has some similarity with the general theory of relativity, where mass warps space-time to create gravitational fields.

\subsection{Fabrication}

Artificial super hydrophobic surfaces were fabricated. These are textures comprising a non-periodic hexagonal lattice of cylindrical Si micro pillars with a certain constant diameter and a variable pitch. Nano-sized geometries appropriately positioned upon the pillars would assure giant SEIRA enhancement. (100) silicon wafers (Jocam, Milan, Italy) were cleaned with acetone and isopropanol to remove possible contaminant and then etched with a $4 \%$ wet HF solution. The wafers were then rinsed with D.I. water and dried with $\mathrm{N}_{2}$. Standard optical lithography techniques (Karl Suss Mask Aligner MA 45, Suss MicroTec GA, Garching, Germany) were employed to dig regular arrays of disks within a layer of positive resist (S1813, Rohm and Haas) that was spin-coated onto clean silicon wafers. Electroless deposition techniques were used to grow silver nanograins within the holes. Upon removal of the residual resist with acetone, a Bosch Reactive Ion Etching (MESC Multiplex ICP, STS, Imperial Park, Newport, UK) process was utilized whereby the final structures were obtained in the form of cylindrical pillars with an aspect ratio greater than two. The electroless grown Ag layer served as mask during the RIE process, while its characteristic granular structure allowed for the enhancement of the SERS/SEIRA signal. The substrates, as a whole, were then covered with a thin (few $\mathrm{nm}$ ) film of a Teflon like $\left(\mathrm{C}_{4} \mathrm{~F}_{8}\right)$ polymer to assure hydrophobicity. The masks necessary for optical lithography were fabricated using standard Electron Beam Lithography (Crestec CABL-9000C Electron beam lithography system) methods, using batch files conveniently generated on the basis of the mathematical rules derived in the former paragraph (Section 2.1).

Electroless deposition is a technique whereby metal ions are reduced and deposited, with the desired size and shape, as metals upon a silicon surface. A detailed explanation of the method goes beyond the purpose of this work, and can be instead found in the recent work [18].

\subsection{SEM characterization}

Several SEM images of the samples were captured to assure reproducibility and repeatability. A dual beam SEM-FIB FEI Nova 600 NanoLab system was used for the measurements. During the acquisitions beam energies of 5 and $15 \mathrm{keV}$, and corresponding electron currents of $0.98 \mathrm{pA}$ and $0.14 \mathrm{nA}$, were used, according to the nature of the sample in analysis. The morphology of the silver nanograins was imaged using the mode two configurations, whereby images can be magnified over $2.510^{6} \times$ and ultra-high resolution may be achieved.

\subsection{FTIR analysis of the Rhodamine deposits}

FTIR microscopic images were recorded using a Nicolet 6700 FT-IR thermo scientific spectrometer, equipped with a MCT-A detector with a high sensitivity. A Continuum Micro FT-IR microscope was used, with a 0.58 N.A., 15X Fixed Cassegrain Objective with a working distance of $15 \mathrm{~mm}$. A high-intensity, ETC. EverGlo mid-IR was used as a source. The interferograms were recorded in reflection-absorption modality with a resolution of $4 \mathrm{~cm}^{-1}$ and were averaged over 100 samples. Each spectrum was base line corrected with a second degree polynomial function after acquisition. Mapping measurements were performed over a region of interest sizing $275 \times 15 \mu \mathrm{m}^{2}$ with a resolution of 36 and 20 points in $x$ and $y$-axis direction, respectively.

\section{Results}

Several SEM micrographs of the SH surfaces were taken over different samples to assess uniformity and reproducibility. In Fig. 1d-e, silicon pillars are arrayed over large areas sizing some hundreds of $\mu \mathrm{m}$ per side, with no or minor defects in their structures which recover a modified hexagonal lattice. This would verify the fabrication process capability to attain extreme control, on a large scale, over the key characteristics of the micropillars such as shape and size, and the distribution of the lattice points in the plane. Those points are distributed as to reproduce a distorted geometry, where the central pillar acts like a large mass that warps the space around, thus attracting the adjacent pillars, in accordance with the contraction function $\chi$ formalized in Eq. (1). On a smaller scale, as in Fig. 1f, the heads of the pillars are decorated with silver nanograin clusters. The image shows that those grains are randomly dispersed with an average grain size of about $50 \mathrm{~nm}$ and a small standard deviation $S_{\mathrm{d}}=20 \mathrm{~nm}$; noticeably, these values are consistent with a local enhancement of the FTIR signal in the order of $10^{6}$ [15].

The most practical property of SH surfaces is a vanishingly small friction coefficient on account of which they can be used for a number of different applications, including, on the medical side, the manipulation and control of diluted solutions [8]. The dynamics of slowly evaporating droplets on micro structured surfaces is very well understood, and is recapitulated in the excellent works [7,1921]. Here, we take advantage of these mechanisms, to induce the collapse of the drop into the desired points in the plane, that is, the transition from the Cassie state [22], where the drop preserves a spherical shape without being pinned to the substrate, to the Wenzel state [23], where instead the drop is impaled by the pillars, and it is stuck to the substrate.

The concentration, manipulation and detection capabilities of the devices were verified using, as a probe, Rhodamine6G (R6G). Solutions were prepared containing R6G molecules with a concentration as low as $10^{-16} \mathrm{M}$. Small drops of this solution were gently positioned upon the substrate; the evaporation process was then followed over time. On account of the reduced friction coefficient of the surface, the drop gradually reduced its contact area until an irreversible transition to a stable state with a low energy content (that is, the Wenzel state), whereby any contact area diminution would prevented.

As a result of the entire process, few molecules were conveniently enforced to confine into a small, well defined area. This area is the region where the substrate is mostly hydrophilic, in close proximity to the contraction fixed point, as defined above. Fig. 2a shows a SEM image of the residual solute of R6G at the final stage of the evaporation process, where the R6G molecules are highly concentrated being accumulated in a bounded region sizing few tens of microns or less. The distribution of the analyte is roughly homogeneous over this small area, and typically includes more than one pillar.

Micro-FTIR mapping measurements were carried out to further substantiate the method. A matrix scan was performed over a rectangular grid as explained in the Section 2 (Fig. 2b). Using the band 
centered at $1660 \mathrm{~cm}^{-1}$ as a reference (Fig. 2c), a 2D raster intensity map of R6G was therefore derived (Fig. 2d), that shows that the FTIR intensity varies on changing the position on the substrate. This scale effect means that the device senses, point by point, a variable signal, that is proportional to the quantity of substance deposited upon the pillars, and notice the strong correlation between the raster and SEM images as in Fig. 2a,d. The rough data points in Fig. $2 d$ were further manipulated. Using a polynomial curve fitting of order three, interpolated FTIR maps were derived with sub-pixel resolution. The contour and 3D plots in Fig. 2e-f, show that the point of maximum intensity coincides with the geometric center of the substrate.

It is important to notice, here, how the volume of the dried residue observed in the SEM image in Fig. 2a is some orders of magnitude larger than what is expected from the very small number of molecules contained within the sample droplet. The mechanisms that can be responsible for an oversized deposit are three, and namely, (i) the presence of external contaminants in the solution, (ii) the presence of extraneous pollutants on the substrate, and (iii) the fabrication process itself, where the chemical inert passivation layer C4F8 (Teflon), deposited upon the pillars for assuring hydrophobicity, can represent, itself, source of external contamination. While the presence of extraneous pollutants can be reduced working in elevated standards of cleanliness, that is, using ultrapure water in a clean room, an excess of Teflon on the surfaces can be cause of concern.

In depositing an let evaporating drops of Milli-Q water not containing any Rhodamine on the super-hydrophobic surface in a clean room (Supplementary Information), it is observed that still a residue is generated on the substrate. Microanalysis (Supplementary Information) shows that the analytical content of such a residue is compatible with a deposit constituted by Teflon which, therefore, is accountable for the discrepancy between the expected and the observed solute residual.

Fortunately, the excess of Teflon does not affect, to appreciable extents, the detection of the analytes, and this is demonstrated by the FTIR results where molecules are sensed in the noticeable low limits of a $10^{-16} \mathrm{M}$ concentration. Nevertheless, future strategies and developments in the field of SH surfaces coupled with SERS/ SIERA devices, necessarily include the realization Teflon free devices. This could be accomplished integrating, upon the pillars, nanoporous silicon matrices. This new, hierarchical structure with a two scale roughness, would exhibit superior hydro-phobicity behavior as shown, for instance, in [11,12].

\section{Conclusions}

Conventional SH surfaces typically comprise micro pillars combined to form a regular hexagonal motif. Here, the pattern of those pillars was conveniently modified to introduce artificial gradients of wettability over the surface. Those gradients generate, in turn, a field of forces that would recall the drop towards a well defined point in the plane upon evaporation. The distribution of points in the plane was correctly designed on the basis of mathematical and physical concepts. The pillars were further decorated with silver nanoparticles aggregates that guarantee superior SERS/SEIRA capabilities. On account of this multiscale nature, the device features different functions or abilities, that are, (i) the concentration of diluted solutions, (ii) the transport and correct positioning of few molecules on a desired region of the device, and (iii) the analysis of those molecules with otherwise unattainable sensitivity.
Here, few molecules of Rhodamine were correctly manipulated, positioned on the active sites of the device, and analyzed using FTIR spectroscopy techniques. This simple concept can be conveniently pursued to foster the realization of advanced bio-sensors of a new generation, with single molecule detection capabilities, for application in a variety of fields, including the large area of early detection of tumors or other diseases.

\section{Acknowledgments}

This work was funded under Project NANOANTENNA FP7HEALTH-2009 (Grant agreement No. 241818), Italian project FIRB 'Rete Nazionale di Ricerca sulle Nanoscienze ItalNanoNet' (cod. RBPR05JH2P-010) and by the EU Commission, the European Social Fund and the Calabria Region (POR Calabria FSE 2007-2013).

\section{Appendix A. Supplementary data}

Supplementary data associated with this article can be found, in the online version, at http://dx.doi.org/10.1016/j.mee.2013.01.036.

\section{References}

[1] G. Das, F. Mecarini, F. Gentile, P. Candeloro, C. Liberale, F. De Angelis, H.M. Kumar, G. Cuda, E. Di Fabrizio, Biosensors and Bioelectronics 24 (2009) 16931699.

[2] F. De Angelis, F. Gentile, F. Mecarini, G. Das, M. Moretti, P. Candeloro, M. Coluccio, G. Cojoc, A. Accardo, C. Liberale, R. Zaccaria, G. Perozziello, L. Tirinato, A. Toma, G. Cuda, R. Cingolani, E. Di Fabrizio, Nature Photonics 5 (2011) 682.

[3] F. Gentile, M. Coluccio, N. Coppedè, F. Mecarini, G. Das, C. Liberale, L. Tirinato, M. Leoncini, G. Perozziello, P. Candeloro, F. De Angelis, E. Di Fabrizio, ACS Applied Materials and Interfaces 4 (2012) 3213-3224.

[4] F. Gentile, G. Das, M. Coluccio, F. Mecarini, A. Accardo, L. Tirinato, R. Tallerico, G. Cojoc, C. Liberale, P. Candeloro, P. Decuzzi, F. De Angelis, E. Di Fabrizio, Microelectronic Engineering 87 (2010) 798-801.

[5] R. Blossey, Nature Materials 2 (2003) 301-306.

[6] A. Lafuma, D. Quéré, Nature Materials 2 (2003) 457-460.

[7] G. McHale, S. Aqil, N. Shirtcliffe, M. Newton, H. Erbil, Langmuir 21 (2005) 11053-11060.

[8] G. McHale, N. Shirtcliffe, M. Newton, Analyst 129 (2004) (2005) 129.

[9] A. Accardo, F. Gentile, F. Mecarini, F. De Angelis, M. Burghamme, E. Di Fabrizio, C. Riekel, Langmuir 26 (2010) 15057-15064.

[10] A. Accardo, F. Gentile, F. Mecarini, F. De Angelis, M. Burghammer, E. Di Fabrizio, C. Riekel, Microelectronic Engineering 88 (2011) 1660-1663.

[11] F. Gentile, A. Accardo, M. Coluccio, M. Asande, G. Cojoc, F. Mecarini, G. Das, C. Liberale, F. De Angelis, P. Candeloro, P. Decuzzi, E. Di Fabrizio, Microelectronic Engineering 88 (2011) 1749-1752.

[12] F. Gentile, M. Coluccio, A. Accardo, G. Marinaro, E. Rondanina, S. Santoriello, S. Marras, G. Das, L. Tirinato, G. Perozziello, F. De Angelis, C. Dorigoni, P. Candeloro, E. Di Fabrizio, Microelectronic Engineering 97 (2012) 349-352.

[13] M. Reyssat, F. Pardo, D. Quéré, Europhysics Letters 87 (2009) 36003.

[14] D.M. Spori, T. Drobek, S. Zurcher, N.D. Spencer, Langmuir 26 (2010) 9465 9473.

[15] R. Aroca, D. Ross, C. Domingo, Applied Spectroscopy 58 (11) (2004) 324A338A.

[16] F. Gentile, E. Battista, A. Accardo, M. Coluccio, M. Asande, G. Perozziello, G. Das, C. Liberale, F. De Angelis, P. Candeloro, P. Decuzzi, E. Di Fabrizio, Microelectronic Engineering 88 (2011) 2537-2540.

[17] B. Pignataro, G. Grasso, L. Renna, G. Marletta, Surface and Interface Analysis 33 (2002) 54-58

[18] F. Gentile, M. Coluccio, A. Toma, E. Rondanina, M. Leoncini, F. De Angelis, G. Das, C. Dorigoni, P. Candeloro, E. Di Fabrizio, Microelectronic Engineering 98 (2012) 359-362.

[19] H. Kusumaatmaja, M. Blow, A. Dupuis, J. Yeomans, Europhysics Letters 81 (2008) 36003.

[20] S. Moulinet, D. Bartolo, European Physical Journal E 24 (2007) 251-260.

[21] M. Reyssat, J.M. Yeomans, D. Quéré, Europhysics Letters 81 (2008) 26006.

[22] A. Cassie, S. Baxter, Trans. Faraday Soc 40 (1944) 546-551.

[23] R. Wenzel, Ind. Eng. Chem. 28 (1936) 988-994. 